\title{
Superior vena cava replacement for lung cancer using a heterologous (bovine) prosthesis: Preliminary results
}

\author{
Lorenzo Spaggiari, MD, PhD, ${ }^{a}$ Domenico Galetta, MD, ${ }^{a}$ Giulia Veronesi, MD, ${ }^{a}$ \\ Francesco Leo, MD, ${ }^{a}$ Roberto Gasparri, MD, ${ }^{a}$ Francesco Petrella, MD, ${ }^{a}$ \\ Alessandro Borri, MD, ${ }^{a}$ Giuseppe Pelosi, MD, and Marco Venturino, MD, ${ }^{\mathrm{c}}$ \\ Milan, Italy
}

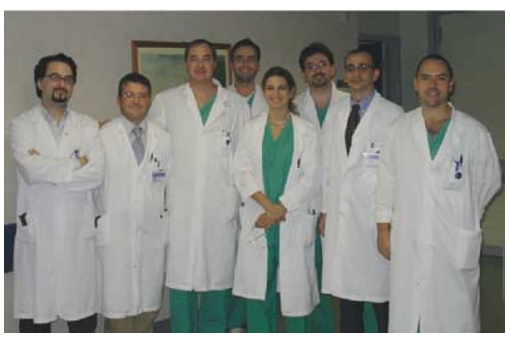

Drs Petrella, Gasparri, Spaggiari, Scanagatta, Veronesi, Galetta, Leo, Borri (left to right)

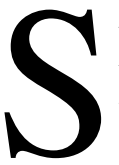

uperior vena cava (SVC) resection for lung cancer is technically feasible, with encouraging oncologic results when modern selection criteria are used. ${ }^{1}$ However, some questions still remain, mainly those regarding which type of prosthetic replacement to use after complete SVC substitution. We successfully experimented with a new revascularization technique for SVC replacement after its complete resection for lung cancer, using a heterologous (bovine) custom-made pericardial prosthesis, and we report the early outcome (oncologic and graft patency results) of a preliminary consecutive series.

\section{Clinical Summary}

From 1998, 70 patients underwent SVC system resection for lung and mediastinal malignancies: 34 (49\%) received prosthetic replacement, and of these, the last 5 patients underwent SVC revascularization with a heterologous (bovine) custom-made pericardial prosthesis.

The surgical approach used was either a large muscle-sparing lateral thoracotomy in the anterior fourth rib section $(n=4)$ or a right hemiclamshell approach $(\mathrm{n}=1)$. After radical lymph node dissection and before lung resection, the SVC was completely removed, and vascular continuity was restored by using the biological prosthesis. A heterologous bovine pericardial patch (No React pericardial patch; Shelhigh, Millburn, NJ; original size, $10 \times$ $20 \mathrm{~cm}$ ) was used (Figure 1,A); it was made tubular with a running 5-0 polypropylene suture (Figure 1, B).

The length of the biological tube used depended on the degree of SVC involvement and was thus variable. The diameter, conversely, was quite standard-approximately $20 \mathrm{~mm}$ after the patch was wrapped around a syringe (Figure 1, $B$ ).

The anastomosis between the heterologous biological prosthesis and the SVC was performed with 5-0 polypropylene sutures (Figure 2). All resections were performed with the crossclamping

\footnotetext{
From the Departments of Thoracic Surgery, ${ }^{a}$ Pathology, ${ }^{\mathrm{b}}$ and Anesthesiology, ${ }^{\mathrm{c}}$ European Institute of Oncology, Milan, Italy.

Received for publication Aug 23, 2005; revisions accepted Aug 26, 2005; accepted for publication Sept 9, 2005.

Address for reprints: Lorenzo Spaggiari, MD, PhD, Department of Thoracic Surgery, European Institute of Oncology, via Ripamonti 435, 20141 Milan, Italy (E-mail: lorenzo.spaggiari@ieo.it).

J Thorac Cardiovasc Surg 2006;131:491-2

$0022-5223 / \$ 32.00$

Copyright $\odot 2006$ by The American Association for Thoracic Surgery

doi:10.1016/j.jtcvs.2005.09.011
}

technique. ${ }^{2}$ Low doses of low-weight heparin were administered after surgery up until 1 month after discharge. All patients were followed up by using a computed tomographic thoracic scan every 3 months.

From October 2003, 5 other consecutive patients underwent operation. All but 1 underwent induction chemotherapy after mediastinoscopy. The mean SVC system clamping time was 31 minutes. Lung resection was performed with a tracheal sleeve pneumonectomy in 4 cases and a right upper lobectomy in the remaining case. One patient died during the postoperative period as a result of pneumonia. The others had uneventful postoperative outcomes, with a mean postoperative hospital stay of 8 days.

During follow-up, 1 patient died at 3 months as a result of evolving disease. Three patients are still alive and disease free (at 21,8 , and 8 months) without signs (on computed tomographic thoracic scan) of late graft thrombosis.

\section{Discussion}

Normally, an autologous pericardial patch is used in cardiac surgery, in reconstruction of the pulmonary artery, and sometimes in the closing of partial vascular defects after SVC or pulmonary artery surgery for lung and mediastinal malignancies. ${ }^{1,3}$ An autologous pericardium is ideal in these situations because of the lower risk of infection and thrombosis compared with a synthetic prosthesis; besides, it does not require anticoagulation therapy. However, an autologous pericardium is not sufficient for creating a long tube after complete SVC resection. In fact, in these situations, SVC replacement with a synthetic (polytetrafluoroethylene) prosthesis is mandatory. The problem with using synthetic prostheses is manifold: the need for prolonged full anticoagulation therapy, the risk of infection (mainly after lung resection), and early graft thrombosis. Plus, little is known about the long-term patency of the synthetic graft after great venous vessel replacement. On the basis of our previous experience with SVC surgery for lung cancer, ${ }^{1,2,4}$ we decided to use biological material instead of a polytetrafluoroethylene graft because of the various risks involved in using a synthetic graft. We choose the bovine pericardial patch because it was experimentally studied in animal models, ${ }^{5}$ which showed long patency of the graft; however, up to now, no human experience concerning SVC replacement with pericardial bovine tube has been reported. Modern biocompatible pericardial patches were introduced recently into clinical cardiothoracic surgical practice. 


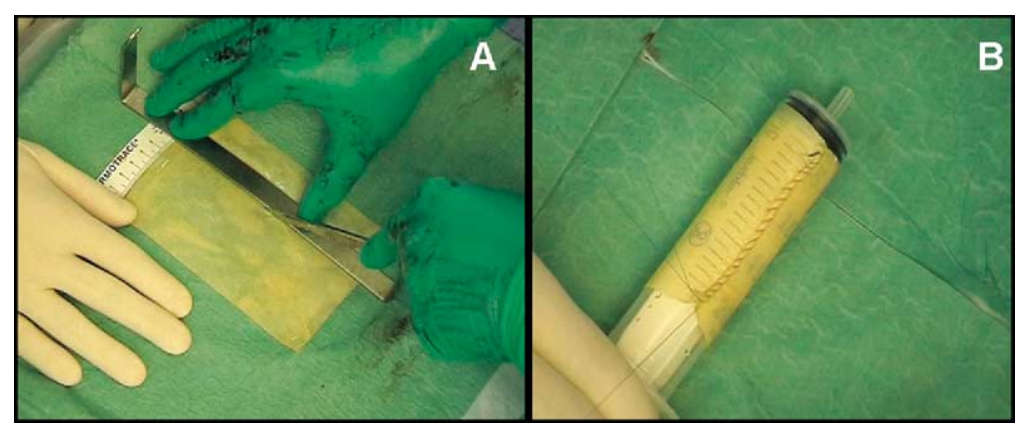

Figure 1. A, Bovine pericardial patch is prepared according to the length of superior vena cava resection. $B$, Heterologous bovine pericardial patch is made tubular by a running 5-0 polypropylene suture.

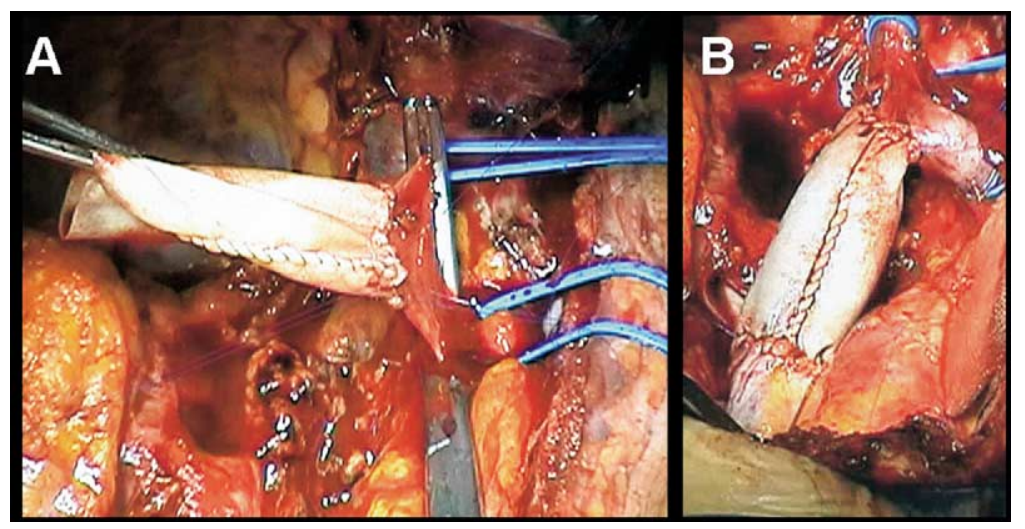

Figure 2. A, Operative picture during superior vena cava (SVC) reconstruction: the proximal anastomosis is in progress. B, SVC replacement with the heterologous pericardial tube is complete.

This patch shows an increased thickness with respect to the human one, it does not shrink, and it retains suture better than fresh pericardium. These patches are totally detoxified, reaching full biocompatibility. These characteristics probably should influence graft patency; in fact, our first patient, still alive and disease free, presented a patent graft at the last follow-up at 21 months after surgery. The other 2 patients, still alive and disease free, have patent grafts at 8 months.

This preliminary experience with 5 consecutive patients confirms that a long tube can be constructed by using a bovine pericardial patch. Current heterologous pericardial tubes are able to retain all the advantages of autologous ones, with no size limits and with excellent results in terms of graft patency.

\section{References}

1. Spaggiari L, Magdeleinat P, Kondo H, Thomas P, Leon ME, Rollet G, et al. Results of superior vena cava resection for lung cancer. Analysis of prognostic factors. Lung Cancer. 2004;44:339-46.

2. Spaggiari L, Thomas P, Magdeleinat P, Kondo H, Rollet G, Regnard JF, et al. Superior vena cava resection with prosthetic replacement for non-small cell lung cancer: long term results of a multicentric study. Eur J Cardiothorac Surg. 2002;21:1080-6.

3. Piccione W, Penfield Faber L, Warren WH. Superior vena cava reconstruction using autologous pericardium. Ann Thorac Surg. 1990;50:417-9.

4. Spaggiari L, Pastorino U. Combined tracheal sleeve and superior vena cava resection for non-small cell lung cancer. Ann Thorac Surg. 2000;70:1172-5.

5. Del Campo C, Love J, Bowes F. Prosthetic replacement of the superior vena cava with custom-made pericardial graft: an experimental study. Can J Surg. 1992;35:305-9. 